\title{
Assessing leadership and clinical performance of pediatric emergency medicine providers during level 1 trauma resuscitations using video review
}

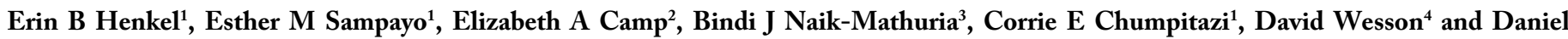 \\ Rubalcava $^{1}$ \\ ${ }^{1}$ Assistant Professor, Department of Pediatrics, Section of Emergency Medicine, Baylor College of Medicine, Texas Children's Hospital, Houston, TX, USA \\ ${ }^{2}$ Doctorate in Statistics, Department of Pediatrics, Section of Emergency Medicine, Baylor College of Medicine, Texas Children's Hospital, Houston, TX, USA \\ ${ }^{3}$ Assistant Professor, Department of Surgery, Division of Pediatric Surgery, Baylor College of Medicine, Texas Children's Hospital, Houston, TX, USA \\ ${ }^{4}$ Professor, Department of Surgery, Division of Pediatric Surgery, Baylor College of Medicine, Texas Children's Hospital, Houston, TX, USA
}

\begin{abstract}
Background: A modified non-technical skills scale for trauma (T-NOTECHS) for assessment of leadership skills in trauma resuscitations has been described in surgical literature. The utility of this tool with Pediatric Emergency Medicine (PEM) providers has not been explored. The objectives of this study were to assess the utility of the T-NOTECHS scoring tool in the pediatric setting and identify barriers to leading a thorough and efficient trauma evaluation.

Methods: We conducted a mixed-methods study utilizing video review and semi-structured interviews of PEM team leaders of Level 1 trauma resuscitations at a quaternary children's hospital. Resuscitation videos were scored using T-NOTECHS by 5 or more reviewers from different disciplines: PEM physicians, pediatric surgeons, and non-physicians including nurses and advanced practice providers (RN/APPs). Median scores were compared using intraclass correlation coefficients (ICC). A sample of subjects scored their own videos and participated in a semi-structured interview. Two coders conducted thematic analysis to identify perceived barriers in leading efficient trauma resuscitations, and member checking was completed.

Results: Thirty trauma resuscitations were reviewed and analyzed; twenty-eight had complete data from all groups. T-NOTECHS scores were highest in the PEM self-review group, followed by surgeons and PEM physicians. RN/APPs scored leaders lowest. There was moderate to substantial ICC agreement between all groups. Four principal themes emerged as perceived barriers to leading efficient trauma resuscitations: communication issues, resource limitations, the role of learners, and a lack of standardization and consistency among providers.

Conclusions: The T-NOTECHS score is a useful tool in evaluating PEM leadership competency during trauma resuscitations and demonstrates reliability amongst scores obtained from reviewer groups with varied trauma experience and training. Systematic interviews of team leaders can identify barriers and means to improve the quality and efficiency of Level 1 trauma evaluations.
\end{abstract}

\section{Introduction}

The leading cause of death in children and young adult ages 1-19 years is unintentional injury; in 2014, over 6400 injury-related deaths were reported in this age group [1]. The American College of Surgeons' (ACS) National Trauma Data Bank 2015 Pediatric Annual Report, notes that during 2015 a total of 143,996 children (ages 0 to 19) were treated at a pediatric trauma center, and of those patients $2,819(1.96 \%)$ died [2]. Studies where trauma resuscitations are led by trauma surgery teams have demonstrated that the presence of a dedicated and welltrained trauma team leader improves patient outcomes and Advanced Trauma Life Support (ATLS) compliance [3]. There are only 28 facilities in the United States and Canada designated as Pediatric-Only Level 1 Trauma Centers; optimizing team leader performance at these facilities may minimize morbidity and mortality for pediatric trauma patients [2].

Training for low-frequency, high-risk events such as major trauma is challenging. Video recording emergency department (ED) resuscitations is an accepted method of evaluating adherence to Advance Trauma Life Support (ATLS) protocols, but limited data exists on using video review to assess team leadership effectiveness. Also, a comprehensive quality improvement program is required for Level 1 Trauma Center certification by the ACS [4]. One way to improve quality is to improve how trauma patients are evaluated and managed in the ED. Different measures and scoring systems have been used to assess efficacy of team leaders and multidisciplinary trauma teams. A checklist initially designed to measure non-technical leadership capabilities in the aviation industry has been modified to evaluate team leaders of trauma resuscitations [5]. This T-NOTECHS rubric, or modified "Trauma NOn-TECHnical leadership Score," has

Correspondence to: Erin B Henkel, Assistant Professor, Department of Pediatrics, Section of Emergency Medicine, Baylor College of Medicine, Texas Children's Hospital, 6621 Fannin St Suite A2210, Houston, TX 77030, USA, Tel: 713-213-7117; E-mail: ebhenkel@texaschildrens.org

Key words: pediatric trauma, leadership, T-NOTECHS, resuscitation, mixed methods

Received: November 17, 2016; Accepted: December 09, 2016; Published: December 14, 2016 
been validated for use in assessing team leadership in adult trauma resuscitations [5]. Studies using this tool have demonstrated that higher leadership scores correlate with better clinical performance, shorter time to disposition, and fewer unreported resuscitation tasks $[5,6]$. Studies evaluating trauma leadership and completeness of trauma resuscitations led by Pediatric Emergency Medicine (PEM) physicians are sparse. The purpose of this study was to objectively assess team leadership of PEM physicians utilizing the T-NOTECHS scoring tool in the pediatric setting, and identify barriers to leading a thorough, complete and timely trauma evaluation.

\section{Methods}

This mixed-methods study was conducted in the ED of an urban quaternary-care children's hospital with Level 1 Trauma Center accreditation by the ACS starting in 2010. In 2015, this ED had an annual census of over 76,000 children ages 0 to 21 -years-old; 12,294 cases of injury per year were seen ( $16 \%$ overall census volume), 276 (2.7\%) of which resulted in full trauma team activation (Level 1 activation). Level 1 trauma activations are based on standard ATLS and ACS guidelines, including vital sign instability for age, low or declining mental status, penetrating injury to the head/neck or torso, and respiratory compromise. The most common mechanism of injury seen at this facility was blunt trauma, including non-accidental trauma. The average length of stay for a trauma patient was 2.7 days, and mortality was $1.4 \%$ of all Level 1 trauma patients seen ( 4 out of 276 patients). The 51 bed ED is staffed with a minimum of two PEM certified physicians, 0-2 PEM fellows, 2 general pediatricians, 2-6 pediatric or emergency medicine (EM) residents, and 0-3 advanced practice providers (APPs) at all times. ED trauma resuscitations are initially lead by PEM-certified physicians and/or PEM fellows with attending supervision. A pediatric surgery attending and/or fellow is expected to be present at the resuscitation within 15 minutes of patient arrival, and takes over as team leader upon arrival. General pediatricians and APPs do not lead Level 1 trauma resuscitations. Upon activation of a Level 1 trauma, responding providers include a minimum of one PEM physician, one PEM fellow if present, one pediatric or EM resident, a pediatric surgery attending and/or fellow, pediatric surgery residents and/or advanced practice providers, respiratory therapists, trauma certified nurses, patient care assistants, a child life therapist, a social worker, and a chaplain. The Institutional Review Board for Baylor College of Medicine approved this study prior to initiation with waiver of informed consent granted for both providers and patients and their families.

\section{Quantitative design and analysis}

Between May 1, 2014 and May 31, 2015, video and audio recordings of Level 1 trauma resuscitations were collected from previously installed automated overhead video recorders in the ED resuscitation room. A convenience sample of 30 videos was selected to capture seasonal variations, a representative sample of a variety of team leaders, and a variety of trauma mechanisms and severity. We excluded videos with inadequate video or audio quality that would prohibit complete review. The recordings were reviewed and scored by a minimum of one PEM provider, one pediatric surgeon, and one trauma RN/APP. All reviewers were ATLS or Trauma Nursing Core Course (TNCC) certified. The videos were reviewed for fifteen minutes, or until the primary and secondary surveys of the trauma evaluation were completed, whichever came first. Each reviewer completed the T-NOTECHS leadership tool (Figure 1), which includes five Likertbased questions that can be analyzed individually and summed.

Descriptive factors were presented using frequencies and percent. Continuous variables were found to be skewed using the Shapiro-Wilk test for normality, therefore medians and interquartile ranges were provided. To assess reviewer consistency among all three groups, the

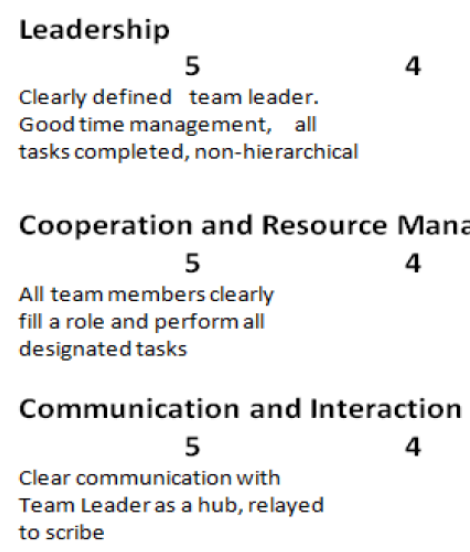

4 designated tasks

\section{Communication and Interaction}

Clear communication with

Team Leader as a hub, relayed to scribe

4

\section{Individual defined, but} some tasks not completed.
Identity of team

\section{Cooperation and Resource Management \\ All team members clearly \\ fill a role and perform all Identity of all members
not clear, some do not
perform assigned tasks}

\section{Assessment and Decision Making}

Orderly and complete $1^{\circ}$

(ABCDE) and $2^{\circ}$ surveys. Plan communicated to team.

4 Communication not always through Team Leader, or not relayed rapidly to scribe

\section{Situation Awareness / Coping with Stress}

5

Untoward findings and

distractions did not upset

systematic and orderly flow.

Team is calm and plans ahead.
4

\author{
Untoward findings caused \\ disruption but did not
} preclude task completion.
2

Unable to discern

role identity of team members
1 Unorganized or incoherent communication on many different levels

1 $1^{\circ}$ and $2^{\circ}$ surveys disorderly and/or incomplete. Plan not clear.

2
1

Untoward findings or interruptions completely upset orderly assessment and task completion Not anticipatory. 
Fleiss' Kappa statistic was chosen and intra-class correlation coefficient (ICC) calculated for paired scores in a post hoc analysis. Interjudge reliability with Fleiss' Kappa and ICC was assessed using a random selection of T-NOTECH scores from each reviewer group. Videos without complete data were excluded. ICC was analyzed using a twoway random model or mixed model with absolute agreement. Singular measure ICC values were reported along with $95 \%$ confidence intervals.

Team leaders in 16 of the trauma resuscitations were asked to selfreview their own videos and complete the same scoring tool (Figure 1). Complete data were available for 15 videos, and ICC was performed comparing the PEM team leader self-scoring to the PEM reviewers' also using random scores for these videos. All analyses were conducted by using the Statistical Package for the Social Sciences (SPSS) version 23 software (IBM Corp., Armonk, NY) and Microsoft Excel 2013.

\section{Qualitative design and analysis}

Semi-structured interviews of a purposive sampling of PEM providers who reviewed and scored their trauma resuscitation videos were conducted and recorded. Audio was entered into NVivo Version 9.0 (QSR International, Doncaster, Victoria, Australia) and analyzed using an iterative coding process to identify principle themes. Researchers used constant comparison analysis, whereby they repeatedly listened to interviews, formulated an initial framework of key codes, and refined these codes with successive reviews. Transcribed notes from interviews were coded by two members (EBH, EMS) of the study team to allow for investigator triangulation. The two members developed mutually agreed-upon definitions for each code and established examples of each code to ensure reliability and trustworthiness. The same two members met to discuss the qualitative findings and develop themes from the codes. Memos of coding decisions were kept to provide consistency in coding as analysis progressed. Member checking was performed to review themes and to check for accuracy and completeness of the findings. The thematic approach is a widely utilized process in the analysis of qualitative data [7]. This approach was used to identify a conceptual framework of themes related to perceived system and provider barriers in the observed setting. Content saturation in the semi-structure interviews was achieved when no new themes emerged in successive interview transcripts, which was found after 16 interviews.

\section{Results}

\section{Quantitative results}

The 22 PEM providers observed in the 30 videos leading trauma resuscitations had a self-reported range of Level 1 trauma experience from 1-12 years, with a median value of $3.5(2.0,8.0)$ years (Table 1$)$. Of the 30 videos reviewed, 28 had complete T-NOTECHS surveys from all three groups of reviewers, of which randomly selected scores were compared (Table 2). Minimum and maximum scores obtainable on this tool are 5 and 25, respectively. Results from the T-NOTECHS

Table 1. Descriptive Characteristics of PEM Providers and Patients from Reviewed Videos.

\begin{tabular}{|c|c|}
\hline Factor & $\mathrm{N}(\%)$ or Median (IQR) \\
\hline \multicolumn{2}{|l|}{ PEM Providers $(\mathrm{N}=22)$} \\
\hline Female providers & $16(72.7 \%)$ \\
\hline Years of trauma experience & $3.50(2.0,8.0)$ \\
\hline \multicolumn{2}{|l|}{ Patients $(\mathrm{N}=\mathbf{3 0})$} \\
\hline Age (years) & $4.0(0.975,10.25)$ \\
\hline Blunt trauma & $24(80.0 \%)$ \\
\hline Died in ED & $3(10.0 \%)$ \\
\hline Died in hospital & $8(26.7 \%)$ \\
\hline
\end{tabular}

$\mathrm{PEM}=$ Pediatric Emergency Medicine; $\mathrm{SD}=$ standard deviation
Table 2. Randomly Selected T-NOTECHS Scores across the Four Rating Groups.

\begin{tabular}{|c|c|c|c|c|}
\hline Video Number & PEM & Surgeon & RN/APP & PEM Self \\
\hline 1 & 16 & 24 & 14 & 23 \\
\hline 2 & 11 & 8 & 7 & 16 \\
\hline 4 & 15 & 20 & 8 & 16 \\
\hline 5 & 25 & 25 & 17 & 23 \\
\hline 6 & 16 & 25 & 11 & 19 \\
\hline 7 & 15 & 14 & 13 & 21 \\
\hline 8 & 19 & 15 & 15 & 22 \\
\hline 9 & 18 & 17 & 19 & 16 \\
\hline 10 & 15 & 21 & 19 & 19 \\
\hline 11 & 6 & 13 & 14 & 15 \\
\hline 12 & 21 & 23 & 23 & 19 \\
\hline 13 & 15 & 15 & 12 & \\
\hline 14 & 25 & 25 & 17 & 21 \\
\hline 15 & 9 & 21 & 5 & \\
\hline 16 & 24 & 21 & 17 & 21 \\
\hline 17 & 19 & 24 & 17 & 23 \\
\hline 18 & 16 & 22 & 21 & 19 \\
\hline 19 & 21 & 25 & 19 & \\
\hline 21 & 23 & 21 & 22 & \\
\hline 22 & 22 & 22 & 24 & \\
\hline 23 & 14 & 16 & 16 & \\
\hline 24 & 15 & 11 & 15 & \\
\hline 25 & 11 & 12 & 15 & \\
\hline 26 & 19 & 18 & 15 & \\
\hline 27 & 19 & 12 & 16 & \\
\hline 28 & 15 & 10 & 17 & \\
\hline 29 & 17 & 21 & 13 & \\
\hline 30 & 12 & 21 & 19 & \\
\hline
\end{tabular}

RN/APP $=$ Registered Nurse and Advanced Practice Providers

analysis are summarized on Tables 3 and 4. In an analysis of Fleiss' Kappa inter-rater reliability between groups on the five sections of the T-NOTECHS tool, there was poor to fair agreement between all three groups ( $\mathrm{k}$ range $=0.03-0.25$ ) with the strongest agreement in the leadership category $(\mathrm{k}=0.25)$ (Table 3$)$. Summed T-NOTECHS scores showed very low agreement $(\mathrm{k}=0.02)$ (Table 3$)$. A post hoc reliability analysis comparing PEM provider training was fair between surgeons and RN/Midlevel (ICC $=0.27$ ) and moderate between PEM and RN/ Midlevel groups $($ ICC $=0.55)$ (Table 4$)$. Self-review by PEM team leaders had a moderate agreement $(\mathrm{ICC}=0.49)($ Table 4$)$.

\section{Qualitative results}

16 PEM team leaders self-reviewed his or her video and completed a semi-structured interview with the primary investigator $(\mathrm{EBH})$. Four principal themes emerged as perceived barriers to leading efficient trauma resuscitations: communication issues, resource limitations, the role of the learner, and lack of standardization (Figure 2). Selected quotes organized by theme can be found in Table 4 .

\section{Communication issues}

Communication barriers were a major theme discussed in all interviews. Concerns included difficulty with handoff from EMS, communication amongst the ED team members, challenges balancing team lead responsibilities, crowd control, and discussions with the surgical teams and/or the pediatric intensive care unit.

With the initial EMS handoff, several providers noted difficulty with "distractions" during this time. "It was hard to listen to the medics [give report]... People do not totally respect the position you're in." 
Table 3. Inter-Rater Reliability of PEM, Pediatric Surgeons and RN/APPs in Evaluating Leadership Performance in the Emergency Department using Randomly Selected T-NOTECHS Scores. $(\mathrm{N}=84)$.

\begin{tabular}{|l|c|c|}
\hline T-NOTECHS Item (Range) & Fleiss Kappa* & 95\% Confidence Interval \\
\hline Leadership (1-5) & 0.25 & $0.01-0.48$ \\
\hline Cooperation and Resource Management (1-5) & 0.08 & $-0.14-0.30$ \\
\hline Communication \& Interaction (1-5) & 0.13 & $-0.11-0.37$ \\
\hline Assessment \& Decision Making (1-5) & 0.03 & $-0.19-0.25$ \\
\hline Situation Awareness/Coping with Stress (1-5) & 0.06 & $-0.17-0.30$ \\
\hline Combined Likert Score (5-25) & 0.02 & $-0.09-0.14$ \\
\hline
\end{tabular}

PEM $=$ Pediatric Emergency Medicine Physicians

RN/APPs $=$ Registered Nurses and Advanced Practice Providers

Table 4. Intra-class Correlations Reliability Analysis Using Paired T-NOTECHS Likert Scores Randomly Selected $(\mathrm{N}=28)$.

\begin{tabular}{|l|c|c|}
\hline Paired Group Comparison & ICC $^{\mathbf{a}}$ & $\mathbf{9 5 \%}$ Confidence Interval $^{\mathbf{a}}$ \\
\hline PEM $v$ s. Surgeon & 0.50 & $0.18-0.73$ \\
\hline Surgeon $v s$. RN/APP & 0.27 & $-0.06-0.56$ \\
\hline PEM $v$ s. RN/APP & 0.55 & $0.23-0.76$ \\
\hline PEM $v$. PEM self & 0.49 & $0.04-0.79$ \\
\hline
\end{tabular}

NOTE: ICC $=$ intraclass correlation coefficient; $\mathrm{PEM}=$ pediatric emergency medicine; $\mathrm{RN}$ $=$ registered nurse APP $=$ advanced practice provider.

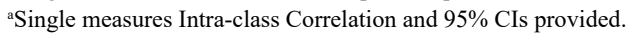

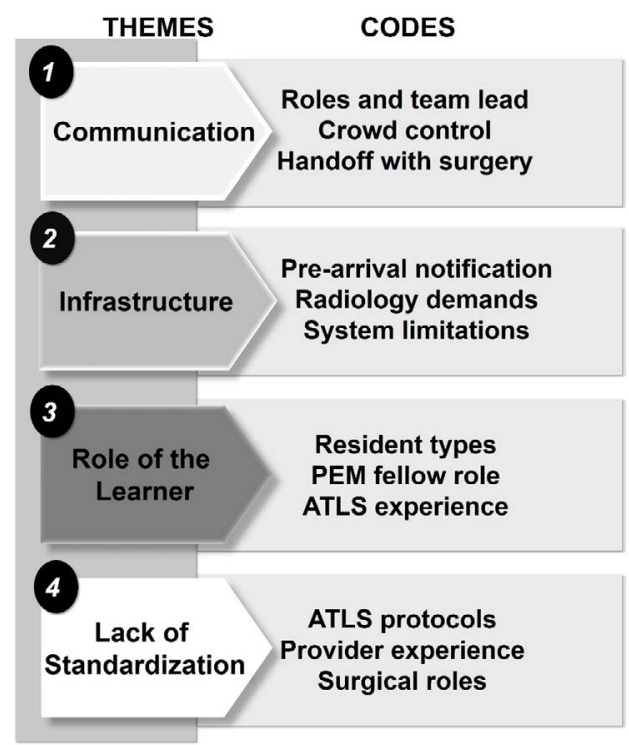

Figure 2. Conceptual Framework of Barriers to Efficient Trauma Resuscitations.

Furthermore, "crowd control" was often cited as a concern. Because "we're a teaching institution" and a have many participants involved in trauma evaluations, "noise levels" and "crowding" were noted as barriers to efficient communication.

Communication concerns with other team members from pediatric surgery, neurosurgery and other surgical subspecialists were noted in all interviews. Later arrival of the surgical team(s) was a frequently identified barrier that contributed to gaps in communication between services.

\section{Resource limitations}

Resource limitations of varying types were discussed in all interviews. Providers in 11 of 16 interviews cited concerns with the existing pre-notification system and variations in the quality and timing of information available to them. Conversely, in traumas that team leaders reflected as going well, they often cited pre-notification as the reason for efficiency and the "key to success."

Another commonly cited resource limitation was lack of or delay in radiology services, noted in 13 of the 16 interviews, such as time required to upload and view imaging sent from transferring facilities, availability and timeliness of X-ray technicians to the bedside, and timeliness of results from CT scans.

Additional system limitations mentioned included readiness of operating rooms and staffing, pediatric intensive care availability, and staffing of the emergency department.

\section{Role of the learner}

Many PEM team leaders, 12 of 16, discussed the role of different types and levels of learners present and participating in the trauma evaluations, including pediatric residents, emergency medicine residents, PEM fellows, surgery residents, and surgical subspecialty residents. Timeliness of ATLS completion as well as thoroughness of primary and secondary survey completion was perceived to be impacted by the learner's previous experience with ATLS and years of training. Quantity of learners present and the role of each individual at bedside was noted as "always challenging." PEM team leaders reported struggling with attempting to balance learning opportunities with efficiency.

The PEM senior fellows interviewed who functioned as team leaders noted occasional difficulty with leadership when they functioned as team lead due to the duality of their role as both leader and learner with the PEM attending also present. Fellows noted feelings of "confusion," a "tug of war" and in some circumstances "flipping" from their role as team leader at some point during the evaluation and management of their patients.

\section{Lack of standardization}

Although ATLS is taught as a standardized process, PEM team leaders in all interviews revealed that they perceive a significant variation between providers, including PEM and surgical practices. Several providers noted specific actions that are frequently missed or take significant time and energy to obtain, including temperature, blood pressure, a fully disrobed exam, and FAST exams. They also stated that some providers have increased "confidence" with trauma, while others who do not may struggle with timeliness and consistency. Additionally, themes centered on the fact that PEM providers often struggle with balancing instincts to minimize invasiveness and diagnostic testing versus strictly following an algorithmic process and ordering more studies and imaging than may be necessary. "We try not to do a lot of stuff... and that puts us in trouble... when you start to second guess the rules."

Given the fact that, in our institution, the surgical services are not physically present in the ED at all times, there was also a theme of potential variation, "conflict," or "discord" in management related to the timing of the pediatric surgery team arrival as well as subspecialty surgical consultants. Individual roles, decision making and transition of care were noted to be most clear and seamless when pre-arrival notification allowed the surgical services to be present at the arrival of the patient and initiation of trauma evaluation.

Member checking with nine PEM physicians and fellows confirmed the above findings. The conceptual framework of codes and themes (Figure 2) was found to be consistent and reproducible. 
Table 5. Select quotes from semi-structured interviews, organized by theme.

\begin{tabular}{|c|c|c|}
\hline Themes & Illustrative Quotes & Interviews $(\mathrm{x} / 16)$ \\
\hline Communication Barriers & & $16 / 16$ \\
\hline Roles and Team Lead & $\begin{array}{l}\text { "We got so off course that there wasn't good closed-loop communication. I don't think I summarized or gave a shared mental model } \\
\text { as I should or normally do. I don't think we verbalized roles well. We got caught up in the stressful not-normal situation and that was a } \\
\text { barrier." } \\
\text { "There were a lot of distractions... It was hard to listen to the medics [give report] while someone else is trying to tell me something else. } \\
\text { People do not totally respect the position you're in, trying to get check out, you often have a lot of distractions... We need to educate one } \\
\text { another... to respect the time [of handoff] and listen to the history." } \\
\text { "I was trying to be team lead, but also talk to mom... to verify code status and limits of the resuscitation. It was challenging because [the } \\
\text { fellow] was focusing on the airway... if you get a second attending in the room to take over... the rest of the ER grinds to a halt." }\end{array}$ & $14 / 16$ \\
\hline Crowd Control & $\begin{array}{l}\text { "At one point we had 3-4 surgeons, } 4 \text { PEM physicians, nursing staff, techs, X-ray techs, a pharmacist, a scribe... there were a lot of } \\
\text { people not actively doing things on the patient who could have taken a step back. This would have helped with noise level." } \\
\text { "People get excited and the noise level is immense. Doing crowd control and being the team lead is difficult... Also needing to } \\
\text { communicate with the family... [Others] can help with this, but eventually the team leader needs to be the face of the person taking care } \\
\text { of their child." }\end{array}$ & $9 / 16$ \\
\hline Handoffs & $\begin{array}{l}\text { "Getting ready to mobilize to the OR was the issue... With surgery, anesthesia and us [PEM] there, the nurse said 'who do we take orders } \\
\text { from?' The transition of care from ER to surgery was fragile... there was some back and forth, confusion... Also with anesthesia, they } \\
\text { wanted blood labs now, then surgery said 'no we don't need those here,' there was back and forth." }\end{array}$ & $16 / 16$ \\
\hline Resource Limitations & & $16 / 16$ \\
\hline Pre-arrival notification & $\begin{array}{l}\text { "The onus should be on the system, not the individuals, to be aware of incoming trauma. We could benefit from a more systematic way of } \\
\text { notifying both [PEM] attendings and fellows. Often it's one and not the other. The overhead page of a trauma arrival is not optimal." } \\
\text { "The key for this trauma running well was pre-notification: both surgery and neurosurgery were present upon arrival, the surgery } \\
\text { attending was right next to me... We had no delay." }\end{array}$ & $11 / 16$ \\
\hline Radiology demands & $\begin{array}{l}\text { "From when the patient arrived to when the CT was uploaded took } 30 \text { minutes or more. At one point neurosurgery and I discussed if we } \\
\text { should just re-scan the head. This delayed care. The second it was uploaded, they said 'go to the OR', but they didn't want to move until } \\
\text { it was available." } \\
\text { "To be a trauma center and not have X-ray in the room, that just seems wrong. In this case we got an airway, couldn't get an X-ray for } 15 \\
\text { minutes. Our radiology [resources] leave more to be desired." }\end{array}$ & $13 / 16$ \\
\hline Infrastructure & $\begin{array}{l}\text { "We need to push ourselves as the physician and push in an interdisciplinary way across the whole trauma system setup to get on point } \\
\text { with getting people to places fast. Cat scan should be ready immediately. A radiologist should be ready immediately to read the scan. } \\
\text { There needs to be a PICU bed or an OR as fast as possible." } \\
\text { "From an efficiency standpoint, we're strapped. We're trying to manage a large volume and high acuity of patients, and take transfer } \\
\text { calls, and cover [trauma patients]... considering having a dedicated trauma team would not be possible in our current staffing situation... } \\
\text { We could still improve notification and comfort level with trauma." }\end{array}$ & $16 / 16$ \\
\hline Role of the Learner & & $12 / 16$ \\
\hline Resident types & $\begin{array}{l}\text { "I think this is intrinsic to teaching institutions and always challenging - when fellows are doing the primary survey it's much more } \\
\text { coherent than when the residents do it. Not so much if it's an EM resident, but the pediatrics residents. They don't always step up to do } \\
\text { the evaluation if they're not comfortable. Maybe this needs more directed teaching with these residents." } \\
\text { "A lot of decisions on the surgery side are made by residents with very little pediatric trauma experience." } \\
\text { "There is this running the information up and down the flagpole with those [on surgical teams] who aren't present." }\end{array}$ & $10 / 16$ \\
\hline PEM fellow role & $\begin{array}{l}\text { "[My role as a fellow as team lead] eventually became nonexistent... in that situation, and in others, once the [attending] starts to take } \\
\text { over, rather than cause that confusion and tug of war, I then quiet down, step back and let them take over... There is a point where I give } \\
\text { it up... I could have asked to continue leading or officially transferred over the role. I've done both." } \\
\text { "That's really when things really got lost. I could have decided to say this is my role [as team lead] or not... At that point the role [of } \\
\text { team lead] flipped... We were doing things in parallel, separately without communicating with each other." }\end{array}$ & $6 / 16$ \\
\hline ATLS experience & $\begin{array}{l}\text { "We should focus on getting the assessment started faster, not just in this case, I think it's a chronic problem. We don't start the } \\
\text { assessment with a pace or urgency commensurate with a Level } 1 \text { trauma center that sees unstable trauma patients... We should be } \\
\text { simultaneously be addressing issues as they are identified." } \\
\text { "We could have used this as a better learning opportunity... No one believes that any further interventions would have saved this child or } \\
\text { changed the outcome... In this situation the greater good is training... and knowing we did everything." }\end{array}$ & $8 / 16$ \\
\hline Lack of Standardization & & $16 / 16$ \\
\hline ATLS protocols & $\begin{array}{l}\text { "The beauty of ATLS is that it's supposed to be so standardized, [but] there's quite a bit of variability within our section. How the timing } \\
\text { of primary and secondary are carried out, the way in which they are verbalized and documented in the trauma nursing documentation." } \\
\text { "An area of confusion is who is going to do [the secondary survey]... if it's time for the secondary survey and we don't see the surgical } \\
\text { team, we don't want to wait... if they come in the middle of the secondary, it's a point of confusion- do we hand it off? Start over?" } \\
\text { "Our FAST exams are improving but there may be some variation in how well we do those... in this case an EM resident performed the } \\
\text { FAST. There are different comfort levels." } \\
\text { "Trauma is so regimented. I'm not saying forget common sense. But if you don't always follow your pathway, there's tendency to miss } \\
\text { something... This is the biggest limitation for pediatric trauma: we try not to do a lot of stuff. That puts us in trouble... when you start to } \\
\text { second guess the rules." }\end{array}$ & $16 / 16$ \\
\hline Provider experience & $\begin{array}{l}\text { "ATLS clearly defines who is to be in charge in various roles. There are PEM providers and fellows who have a varied experience with } \\
\text { pediatric trauma compared to the surgeons. There is a personality of provider that certain traumas run seamlessly and effortlessly. Some } \\
\text { of the changes in decision making when surgery arrives later, and depending on who was initially running the trauma, is a communication } \\
\text { issue. It changes based on provider." }\end{array}$ & $12 / 16$ \\
\hline Surgical roles & $\begin{array}{l}\text { "The biggest challenge is making sure consults are available - not calling if they are not needed, but getting them at the bedside quickly } \\
\text { if we do [need them]." } \\
\text { "The goal is to streamline... the balance of the balance of transition between [PEM and surgery] team leaders... If surgery arrives } \\
\text { later and we have already completed primary and secondary, they often repeat it even if the results are communicated. I understand } \\
\text { thoroughness, but sometimes the two teams can run in parallel and not in tandem... If surgery is present from the beginning, those roles } \\
\text { are more clearly delineated." }\end{array}$ & $16 / 16$ \\
\hline
\end{tabular}




\section{Discussion}

This is the first known study assessing the T-NOTECHS tool in the pediatric-specific setting and demonstrating its validity and reliability, with moderate agreement between PEM physicians and surgeons, as well as between PEM physicians and RN/APP groups. These are three groups with diverse training and experience in caring for pediatric trauma patients. We expected the PEM scorers to give the highest scores to their peers whose performance they were assessing; but, we found instead that the surgeon group rated the PEM providers with higher T-NOTECHS scores. As reported in other studies with selfreview, the self-scoring group rated themselves highest, demonstrating a bias towards stronger performance when reviewing their own videos.

The surgeon group scored the PEM team leaders highest of the three independent groups, while the nursing and APP group scored them the lowest. This raises the question of why the RN/APP group felt that leadership was lacking. We presume that the perception of quality of leadership is largely based on the training and experience of the reviewer, in this case three reviewer groups with very different training in caring for pediatric trauma. The surgeon group may be focusing on efficiency, speed and completeness of the ATLS protocols, while nursing may be more focused on communication skills and overall logistics of completing the trauma survey in an orderly fashion. These findings differ from a prior published study comparing nursing scores to physician scores, in which scores were discordant, but nursing scores were higher in all categories except leadership on the T-NOTECHS tool [8]. Further studies in assessing leadership qualities between different disciplines involved in trauma resuscitations are warranted.

\section{Limitations}

These results are limited to 28 Level 1 trauma evaluations over a 13-month period. While we attempted to select the largest variety of team leaders and cover all seasons, this is not fully representative of the leadership skills of all 40+ PEM faculty practicing within our section. Some videos were excluded due to poor audio and/or visual data limiting review, and additionally there was a 1.5 -month period during which no videos were available due to software barriers and audiovisual hardware upgrade in the ED. We may have found more variability in performance if we were able to include evaluations of all PEM providers in the section.

The reviewers scoring the videos, although not present in the specific videos, nevertheless work with and know well all the providers reviewed, and thus may demonstrate some bias in scoring based on their background knowledge and prior experiences with each provider. Due to the institutional rules on internal video reviews for resuscitations, we were not able to include completely anonymous reviewers with no personal knowledge of the study subjects (PEM physicians).

\section{Future directions}

PEM physicians identified multiple barriers that they felt impeded efficiency and timeliness during trauma evaluations. There have been a number of system changes and improvements throughout the first six years of Level 1 trauma certification to address these issues and improve the process, specifically addressing the notification process, radiology services, learner roles, and standardized protocols. PEM physicians involved in the video review for this study, as well as a subsequent quality improvement project involving ongoing video review of Level 1 trauma evaluations, has improved awareness amongst the group regarding the importance of clear and concise leadership during these evaluations. Many PEM providers have remarked how video review alone has affected their attitudes and behaviors during trauma resuscitations.

Future studies utilizing the T-NOTECHS tool in the pediatric setting would help to further assess the utility of the score in resuscitations. While developed for trauma, this tool could be easily generalizable to all types of resuscitations, medical and trauma. Utilizing this tool for severely injured trauma patients in conjunction with video review has the potential to be used for directed physician feedback and ongoing continuing medical education, quality improvement, teaching of residents and fellows, and in simulation.

\section{Authors contribution}

EBH: Literature search, study design, data collection, data analysis, data interpretation, writing, critical revision

EMS: Literature search, study design including qualitative content expert, data collection, data analysis, data interpretation, writing, critical revision

EAC: Data analysis, data interpretation, critical revision

BJNM: Study design, data collection, critical revision revision

CEC: Study design, data collection, data interpretation, critical

\section{DW: Data collection, critical revision}

DMR: Literature search, study design, data collection, data analysis, data interpretation, writing, critical revision.

\section{References}

1. Center for Disease Control and Prevention. WISQARS ${ }^{\mathrm{TM}}$ : Leading Causes of Death Reports, National and Regional, 1999-2014.

2. American College of Surgeons. National Trauma Data Bank 2015 Pediatric Annual Report.

3. Santora TA, Trooskin SZ, Blank CA, Clarke JR, Schinco MA (1996) Video assessment of trauma response: Adherence to ATLS protocols. American Journal of Emergency Medicine 14: 564-569. [Crossref]

4. American College of Surgeons (2014) Committee on Trauma. Resources for Optimal Care of the Injured Patient.

5. Steinemann S, Berg B, DiTullio A, Skinner A, Terada K, et al., (2012) Assessing teamwork in the trauma bay: Introduction of a modified "NOTECHS" scale for trauma. American Journal of Surgery 203: 69-75. [Crossref]

6. Pucher PH, Aggarwal R, Batrick N, Jenkins M, Darzi A (2014) Nontechnical skills, performance and care processes in the management of the acute trauma patient. Surgery 155: 902-929. [Corssref]

7. Ezzy D (2002) Qualitative Analysis: Practice and Innovation.London: Routledge.

8. Steinemann S, Kurosawa G, Wei A, Ho N, Lim E, et al., (2016) Role confusion and self-assessment in interprofessional trauma teams. The American Journal of Surgery 211: 482-488. [Crossref]

Copyright: (C2016 Henkel EB. This is an open-access article distributed under the terms of the Creative Commons Attribution License, which permits unrestricted use, distribution, and reproduction in any medium, provided the original author and source are credited. 